\title{
Unilateral peak nasal inspiratory flow, normal values in adult population*
} \author{
Alberto Staffieri' ${ }^{1}$ Valerie J. Lund ${ }^{2}$ \\ Department of Neurosciences, Otolaryngology Section, University of Padova, Padova, Italy \\ Ear Institute, University College London, London, United Kingdom \\ Department of Statistical Sciences, University of Padova, Padova, Italy
}

Giancarlo Ottaviano', Glenis K. Scadding², Bruno Scarpa³ ${ }^{3}$ Daria Accordi',
Rhinology 50: 386-392, 2012

DOI:10.4193/Rhino.12.071

*Received for publication:

April 25, 2012

Accepted: September 1, 2012

\section{SUMMARY}

Aims: Measurement of Peak Nasal Inspiratory Flow (PNIF) is a cheap, simple, easily performed method to assess nasal patency and it is suitable for serial measurements and for home use. The purpose of this study was to establish normative unilateral PNIF data for a healthy adult population and provide charts relating unilateral PNIF normal values with various explanatory variables.

Methods and results: Repeated measurements of PNIF and unilateral PNIF were performed in 109 volunteers. Ninety seven of these fulfilled the study criteria and all of them were non-smokers, non-asthmatic, without nose and paranasal sinus problems, with ages ranging from 13 to 80 years. Data were statistically analysed and tables were produced relating unilateral PNIF to height which was the only studied variable that correlated statistically with unilateral PNIF.

Conclusions: The measurement of unilateral PNIF, providing the present data are confirmed in a larger series, could be a useful method to study single nostril patency to aid diagnosis of nasal disease, especially when it is necessary to assess the functional effects of unilateral nasal septal deviations or in all cases where there is a suspicion of a unilateral nasal occlusion. This pilot study provides initial normative unilateral PNIF data.

Key words: Peak Nasal Inspiratory Flow, unilateral Peak Nasal Inspiratory Flow, nasal patency, normal values

\section{Introduction}

Nasal airway obstruction is a common problem in ENT practice and has been shown to correlate with decreased quality of life as a result of, amongst others, decreased quality of sleep, (chronic) rhinosinusitis, otitis media and asthma ${ }^{(1)}$.

The measurement of nasal patency is of considerable importance for rhinologists and respiratory physiologists. Nowadays, rhinomanometry (RM) is regarded as the benchmark for the measurement of nasal airway resistances ${ }^{(2)}$. Although RM is the gold standard for the assessment of nasal resistance, peak nasal inspiratory flow (PNIF) has been shown to be highly correlated with nasal airway resistances, reproducible in the evaluation of nasal airway obstruction and as good an indication of objective nasal patency as formal rhinomanometry ${ }^{(3)}$. Moreover, PNIF is a cheap, simple, easily performed method to assess nasal patency and it is suitable for serial measurements and for home use ${ }^{(4)}$.

In the recent past, normal PNIF values both for adult and paediatric populations have been published by many authors (5-11) allowing the application of this technique to the results of septoplasty ${ }^{(4)}$. Unfortunately, all the published data on PNIF values has been obtained by testing both nostrils at the same time, despite the fact that a knowledge of unilateral nasal patency is often required, for example in selecting patients for surgery ${ }^{(4)}$. This is particularly relevant when more than $17 \%$ of the patients are unable to determine the correct side of their nasal obstruc- 
tion ${ }^{(12)}$, or when studying unilateral nasal valve stenosis. In this pilot study, the authors have tried to establish baseline normal values of unilateral PNIF in adult subjects.

\section{Materials and methods}

Study design

A diverse population of 109 subjects ranging from 13 to 80 years old was recruited at the Department of Otolaryngology, Head and Neck Surgery of Padova University (from colleagues, nurses, patients attending for problems other than the nose and from patients' relatives). On enrolment into the study, all subjects were asked to complete a SNOT 22 questionnaire ${ }^{(13)}$. They were also asked if they were experiencing nasal blockage or any other nasal problem, if they were smokers, asthmatic or had undergone any previous surgery on the nose and paranasal sinuses. All the subjects with a score $<1$ on the SNOT 22, who were non-smokers, non-asthmatic and without any previous sinonasal surgery were asked their age, race and medications used and had their height measured. Of the 109 subjects, 12 women were excluded from the study as they were taking oral contraceptives. Ninety seven volunteers were finally entered into the study, none of whom took any medication, such as $\beta$-blockers or corticosteroid, which could have affected nasal patency. The present investigation was conducted in accordance with the 1996 Helsinki Declaration and was approved by the internal committee of the Section.

\section{Measurements}

A portable Youlten peak flow meter (Clement Clark International) was used for the measurement of PNIF. The masks attached to the spirometer were chosen to fit tightly on each subject's face without touching the nose and were cleaned with swabs saturated with alcohol (Sterets, Seton Healthcare Group pcl) and dried between every subject tested.

All subjects were tested while sitting and were encouraged to inhale as hard and fast as they could through the mask keeping the mouth closed and starting from the end of a full expiration as previously described ${ }^{(5,6)}$. Three satisfactory maximal inspirations were obtained and the greatest of the three results was taken as the PNIF. After that, using adhesive tape (Microfoam; $3 \mathrm{M})$ to seal off one nostril at a time, PNIF was measured from the other unsealed nostril three times and again the greatest result was taken respectively, from the left (IPNIF) and from the right (rPNIF).

\section{Statistics}

Statistical analysis was undertaken with the objective of obtaining a model relating the variable IPNIF and rPNIF to the various exploratory variables available. All analyses were based on standard analysis of variance tests. The tests comprised comparisons of the ratio of mean regression and mean residual sums of squares to an $\mathrm{F}$ distribution with appropriate degrees of freedom. Generally, we took $5 \%$ as the critical level of significance in our tests. We also used standard residual and probability plots to verify the adequacy of the Normality assumption in our models. We selected significant variables by a stepwise backward procedure based on reduction of the Akaike information criterion (AIC) ${ }^{(14)}$.

\section{Results}

Mean and standard deviation of the available variables are shown in Table 1 separately for males and females. The age distribution of the population is presented in Table 2.

To compare these results with those from a previous study ${ }^{(5)}$ where the objective was to obtain a model relating the variable PNIF to age, sex and height, the same analysis was conducted on the present data and PNIF values shown to be in line with those previously reported, indicating good data quality.

Figure 1 shows PNIF plotted against age for male and female subjects confirming a general diminution of PNIF with age and a slight difference between the two sexes, albeit with a large residual variability, similar to that reported in our previous experiences ${ }^{(5,6)}$.

We reduced the heterogeneity in variability by taking the transformation MODPNIF $=(\text { PNIF })^{1 / 2}$. A plot of this variable against age for each sex is shown in Figure 2. In this figure mean PNIF estimates for subjects for respective heights 160,170, 180 and $190 \mathrm{~cm}$ for males and 150,160,170 and $180 \mathrm{~cm}$ for females were included. The model used for the present data analysis was

MODPNIF $=b_{0}+b_{1} A G E+b_{2} I_{S E X}+b_{3} H E I G H T+e$

in which $I_{S E X}$ is an indicator variable that takes the value of 1 for male subjects and 0 for female subjects, and $e$ is a Normal random variable. From the model estimate summary shown in the first part of Table 3 it is possible to see that age is significant ( $p=0.02$, with a power of 0.42 ), while sex and height are marginally significant $(p=0.1)$. Studying the relationships between the explanatory variables, we observed that sex and height were strongly related $\left(h^{2}=0.39, \mathrm{p}<0.001\right)$, age and height had a weak negative correlation $(r=-0.25, \mathrm{p}=0.008)$, and age and sex were not related $\left(h^{2}=0.001, \mathrm{p}<0.66\right)$ indicating again good data quality.

As the results were similar to what had been expected, we proceeded with the statistical analysis both on IPNIF and rPNIF results. In both cases we applied the same transformation: IMODPNIF $=(I P N I F)^{1 / 2}$ and $r M O D P N I F=(r P N I F)^{1 / 2}$. Table 3 also shows model estimate summaries for left and right nostrils, respectively. 
Table 1. Mean IPNIF, rPNIF and PNIF values in males and females.

\begin{tabular}{|c|c|c|c|c|}
\hline & \multicolumn{2}{|c|}{ Males $(n=52)$} & \multicolumn{2}{|c|}{ Females $(n=45)$} \\
\hline Variable & Mean & SD & Mean & SD \\
\hline Age (years) & 42.8 & 15.65 & 42.43 & 16.77 \\
\hline Height (cm) & 177.2 & 8.51 & 164.3 & 7.38 \\
\hline IPNIF(L/min) & 111.8 & 42.92 & 86.74 & 29.89 \\
\hline rPNIF (L/min) & 107.8 & 35.61 & 87.44 & 31.07 \\
\hline PNIF (L/min) & 158.1 & 44.77 & 126.7 & 36.09 \\
\hline
\end{tabular}

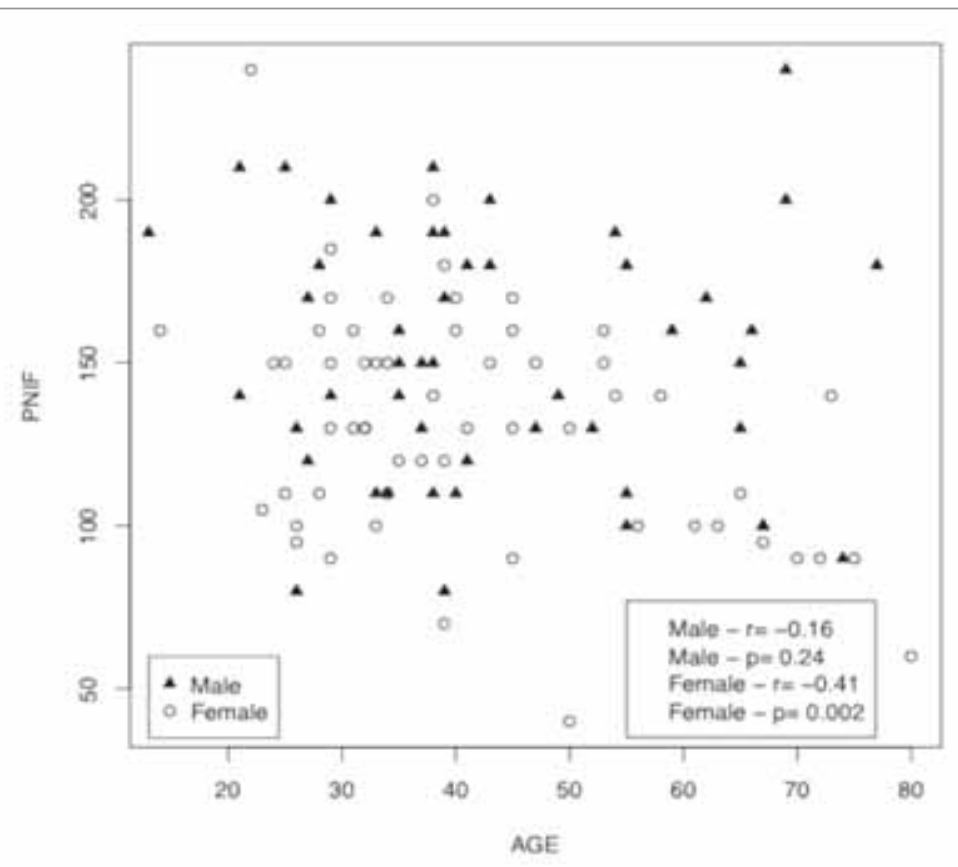

Figure 1. PNIF against age, for male and female subjects.

Only height is significant in the models, its effect is similar for both left and right nostrils and it is higher than when both nostrils are considered together. By using these models we plotted the mean expected values of rPNIF and IPNIF at specified heights with relative confidence intervals (Figure 3). We calculated these values again by using the inverse transformations $I P N I F=I M O D P N I F^{2}$ and $r P N I F=r_{M O D P N I F}{ }^{2}$. As described in the Appendix, expected values of rPNIF and IPNIF are obtained as well as approximate variances, from which we obtained approximated confidence intervals.

A further analysis was conducted to compare both IPNIF and rPNIF with PNIF values. Figure 4 shows these comparisons and the plot of PNIF with the sum of IPNIF and rPNIF. The line in the plots indicates when the two quantities are equal. As expected, most of the subjects show a larger nasal flow when using both nostrils, even if in a very small portion of cases (about 2 or $3 \%$ ) unilateral PNIF (either IPNIF or rPNIF) is higher than PNIF. Finally, IPNIF and rPNIF sum value was greater than PNIF obtained by testing both nostrils at the same time, even if about $10 \%$ of subjects showed a different behaviour. A model to predict the sum of the two nostrils PNIF has been fitted to data (last part of Table 3). Once again, height was the only significant variable.

\section{Discussion}

Rhinomanometry is a well-established method to assess nasal airway resistance. It is an acceptable and safe method to assess nasal airway obstruction, but it is time-consuming, needs experience, is not easily transportable and the equipment is rather expensive.

The use of a reliable, cheap and simple method for assessing nasal airway obstruction is highly desirable and in the last few years a number of researchers have concentrated their work on PNIF with the purpose of defining normal values. 
Table 2. Number of volunteers for each age group.

\begin{tabular}{|cc|}
\hline Classes of age (years) & Number of volunteers \\
\hline $10-20$ & 2 \\
\hline $20-30$ & 26 \\
$30-40$ & 36 \\
$40-50$ & 15 \\
$50-60$ & 11 \\
$60-70$ & 13 \\
$70-80$ & 6 \\
\hline
\end{tabular}

Table 3. Model estimates summaries.

\begin{tabular}{|c|c|c|c|c|}
\hline \multicolumn{5}{|l|}{ PNIF } \\
\hline & Estimate & Std. Error & $t$ value & $\operatorname{Pr}(>[t])$ \\
\hline Intercept & 6.484 & 3.733 & 1.736 & 0.085 \\
\hline AGE & -0.025 & 0.011 & -2.287 & 0.024 \\
\hline SEXM & 0.691 & 0.415 & 1.665 & 0.099 \\
\hline HEIGHT & 0.036 & 0.021 & 1.663 & 0.099 \\
\hline \multicolumn{5}{|l|}{ Right nostril } \\
\hline & Estimate & Std. Error & t value & $\operatorname{Pr}(>[t])$ \\
\hline Intercept & -1.972 & 3.148 & -0.626 & 0.532 \\
\hline HEIGHT & 0.070 & 0.018 & 3.807 & $<0.001$ \\
\hline \multicolumn{5}{|l|}{ Left nostril } \\
\hline & Estimate & Std. Error & $\mathrm{t}$ value & $\operatorname{Pr}(>[t])$ \\
\hline Intercept & -1.760 & 2.866 & -0.614 & 0.540 \\
\hline HEIGHT & 0.068 & 0.017 & 4.051 & $<0.001$ \\
\hline \multicolumn{5}{|c|}{ IPNIF and rPNIF sum } \\
\hline & Estimate & Std. Error & $t$ value & $\operatorname{Pr}(>[t])$ \\
\hline Intercept & -2.503 & 3.796 & -0.659 & 0.511 \\
\hline HEIGHT & 0.097 & 0.022 & 4.372 & $<0.001$ \\
\hline
\end{tabular}

Recently some authors have pointed out that a limitation of using PNIF in clinical practice, is the lack of normative data for the single nostril. This severely limits the usefulness of PNIF in those conditions where it is important to measure the airflow for each side of the nose separately, for example when selecting patients with unilateral nasal septum deviation for septoplasty (4).

The present study confirms that the effect of age on PNIF is significant while sex and height are only marginally significant. However, in studying single nostril PNIF results (IPNIF and rPNIF), intriguingly we have found that age is not significant to the model. This means that, whilst PNIF decreases with age, both IPNIF and rPNIF do not significantly change with age. Moreover,
PNIF in males is higher than in females, while IPNIF and rPNIF seem not to change significantly between sexes. These results could be due to the reduced amount of airflow associated with unilateral PNIF actuations as clearly shown in Figs $4 \mathrm{a}$ and $4 \mathrm{~b}$. The only variable significantly related to both IPNIF and rPNIF was height. From the study of Nunn and Greg ${ }^{(15)}$ we know that pulmonary peak expiratory flows are strictly related to patients' height. Furthermore a relatively recent study demonstrated a correlation between pulmonary airflow and nasal airflow ${ }^{(6)}$. It seems that, when measuring unilateral PNIF, because the effects of age and in part of gender are largely lost, the effect of height becomes the most important variable in the model. This con- 


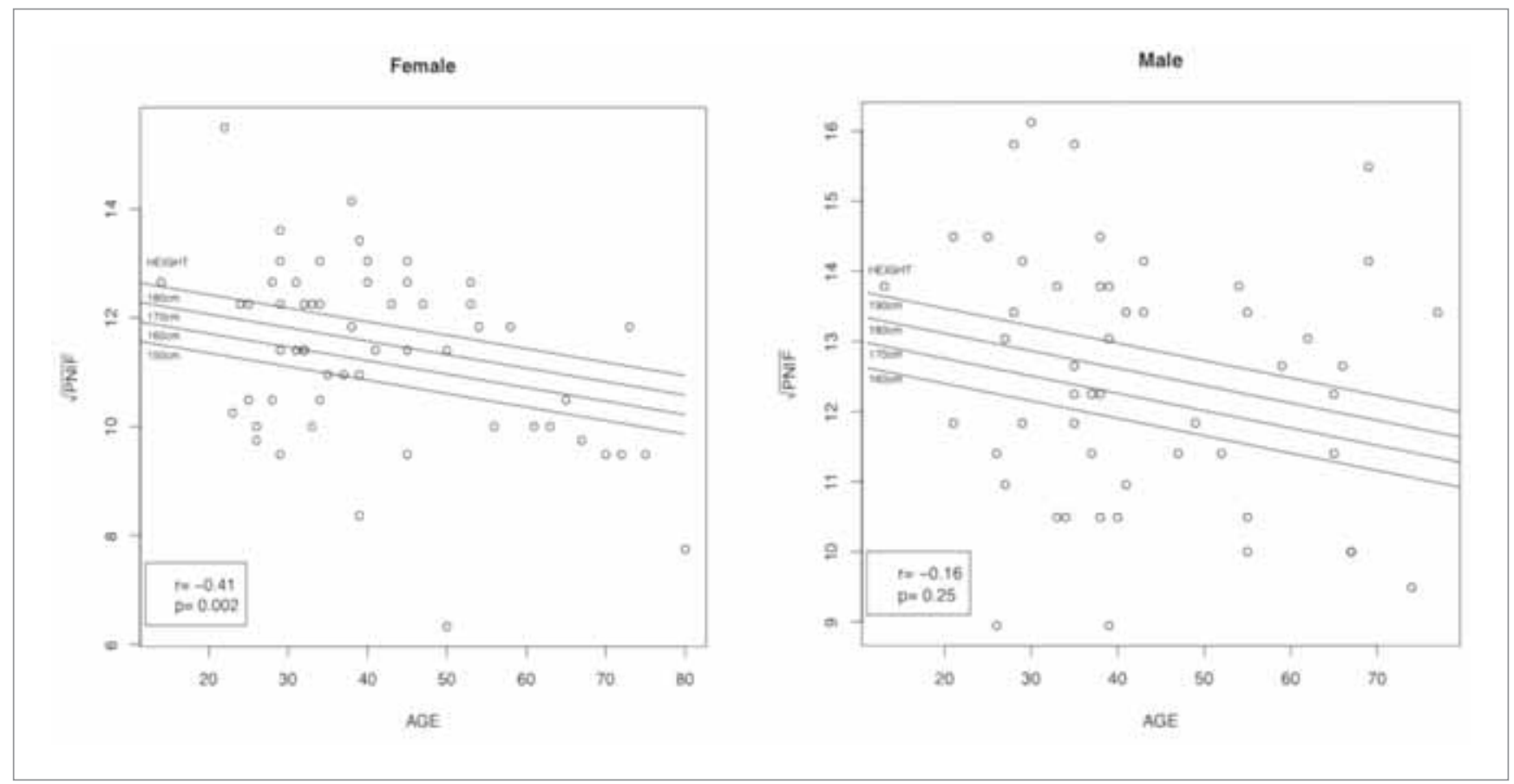

Figure 2. MODPNIF against age, for male and female subjects.

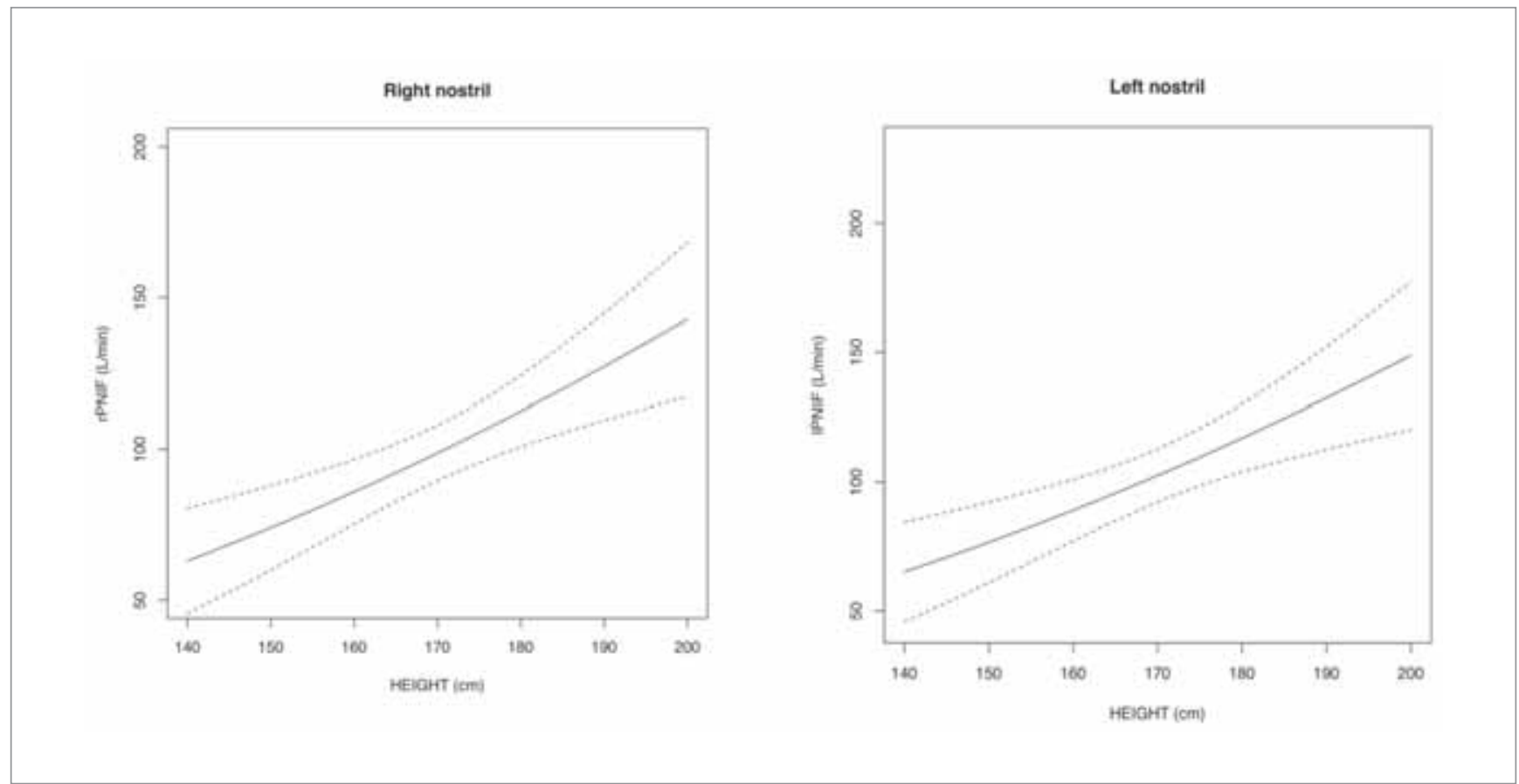

Figure 3. Mean estimates of rPNIF and IPNIF for specified height. The curves were obtained by fitting a model to data and show a highly significant correlation of $0.36(p<0.0001)$ for rPNIF and $0.34(p<0.0001)$ for IPNIF.

firms that nasal airflow in particular when tested unilaterally, is strictly related to pulmonary volumes and thereafter to patients' height.

The sum of IPNIF and rPNIF is higher than when PNIF is measured bilaterally (Figure 4c) which is probably due to the fact that, when testing a single nostril at a time, applying the Venturi principle, the amount of airflow (unilateral PNIF) that enters the nostril is higher than that which enters the same nostril when testing both nostrils at the same time (PNIF).

We conclude that the measurement of unilateral PNIF could be useful for rhinologists to assess single nostril patency and to 


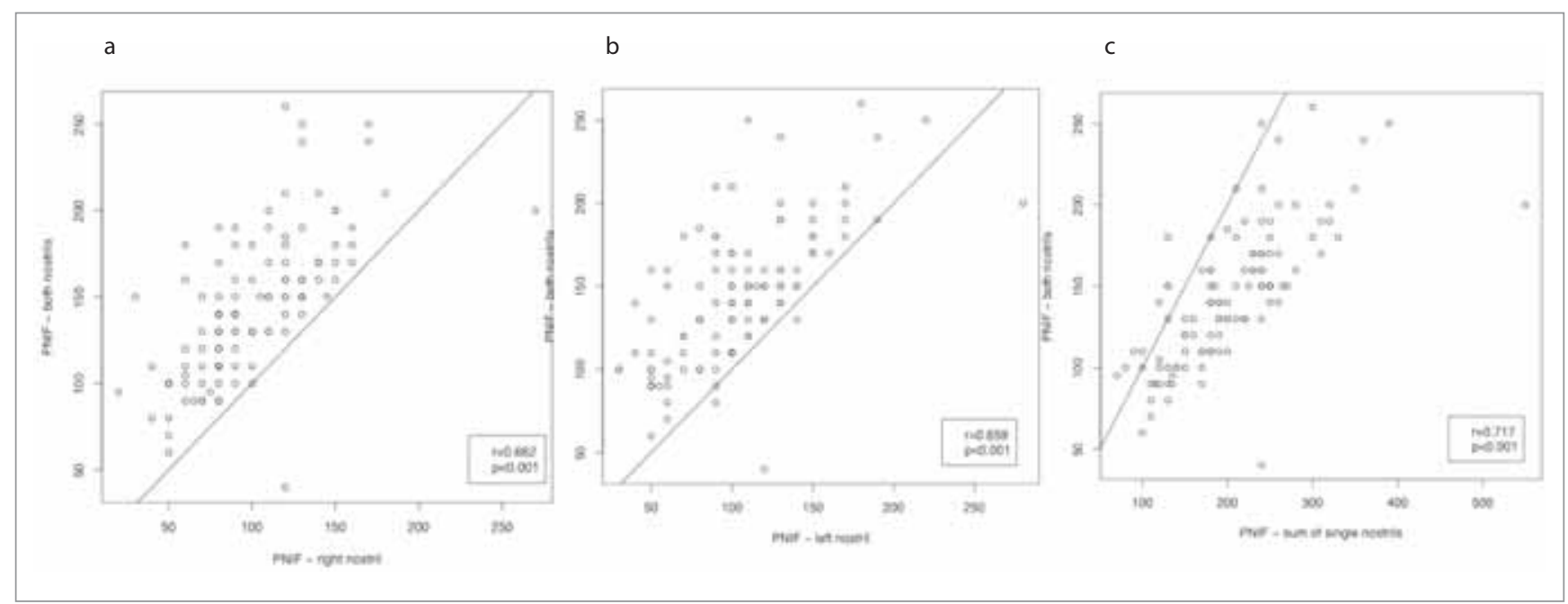

Figure 4. a) PNIF and rPNIF; b) PNIF and IPNIF; c) PNIF and rPNIF + IPNIF sum. .

compare it with total nasal patency. In future, unilateral PNIF should be studied in patients with unilateral or bilateral nasal obstruction in order to know whether unilateral PNIF is sensitive to detect various degrees of nasal obstruction. If the present results are confirmed in a larger series of healthy volunteers and obstructed patients, the measurement of unilateral PNIF could become an easy method to assess septal deviation or any case where there is suspicion of single nostril occlusion. We believe that the Tables presented are an important first step to obtain a reference for normal IPNIF and rPNIF ranges for the study of nasal patency, especially when selecting patients for surgery.

\section{Conflict of interest}

None

\section{Appendix. Statistical analysis}

To try to reduce the heterogeneity in variability, we considered the Box-Cox family of transformations, which tended to suggest the square root transformation for all the considered variables: MODPNIF $=(P N I F)^{1 / 2}, I M O D P N I F=(I P N I F)^{1 / 2}$ and $r M O D P N I F=$ $(\text { rPNIF })^{1 / 2}$.

The model used for the present data analysis was

$$
\text { MODPNIF }=b_{0}+b_{1} \text { AGE }+b_{2} \text { ISEX }+b_{3} \text { HEIGHT }+e
$$

in which ISEX is an indicator variable that takes the value of 1 for male subjects and 0 for female subjects, and e is a Normal random variable.

The expected value of rPNIF and IPNIF are

$E(r P N I F)=\{E(r M O D P N I F)\}^{2}+\operatorname{var}(r M O D P N I F)$ and

$E(I P N I F)=\{E(I M O D P N I F)\}^{2}+\operatorname{var}(I M O D P N I F)$

respectively, which are easily obtained by the least squares results.

Delta method has been used to approximate variances of rPNIF and IPNIF, from which we get approximated confidence intervals

$$
\operatorname{Var}(\text { rPNIF }) \approx 4\{\mathrm{E}(\mathrm{rMODPNIF})\}^{2 *} \operatorname{var}(\text { MMODPNIF })
$$

and

$$
\operatorname{Var}(\text { IPNIF }) \approx 4\{\mathrm{E}(\text { IMODPNIF })\}^{2 *} \operatorname{var}(\text { IMODPNIF). }
$$




\section{References}

1. Corey JP, Houser SM, Ng BA. Nasal congestion: a review of its etiology, evaluation, and treatment. Ear Nose Throat J. 2000; 79: 690-693.

2. Clarke RW, Jones AS, Richardson H. Peak nasal flow- the plateau effect. J Laryngol Otol. 1995; 109: 399-402.

3. Holmström M, Scadding GK, Lund VJ, Darby YC. Assessment of nasal obstruction. A comparison between rhinomanometry and nasal inspiratory peak flow. Rhinology. 1990; 28: 191-196.

4. Holmstrom M. The use of objective measures in selecting patients for septal surgery. Rhinology 2010; 48: 387-393.

5. Ottaviano G, Scadding GK, Coles S, Lund VJ. Peak nasal inspiratory flow. Normal range in adult population. Rhinology. 2006; 44 32-35.

6. Ottaviano G, Lund VJ, Coles S, Staffieri A, Scadding GK. Does peak nasal inspiratory flow relate to peak expiratory flow? Rhinology. 2008; 46: 200-203.

7. Papachristou A, Bourli E, Aivazi D, et al. Normal peak nasal inspiratory flow rate values in Greek children and adolescents.
Hippokratia. 2008; 12: 94-102.

8. Klossek JM, Lebreton JP, Delagranda A Dufour X. PNIF measurement in a healty French population. A prospective study about 234 patients. Rhinology. 2009; 47: 389-392.

9. Bouzgarou MD, Saad HB, Chouchane A, et al. North African reference equation for peak nasal inspiratory flow. J Laryngol Otol. 2011: 125: 595-602.

10. da Cunha Ibiapina C, Ribeiro de Andrade C, Moreira Camargos PA, Goncalves Alvim C, Augusto Cruz A. References values for peak nasal inspiratory flow in children and adolesccent in Brazil. Rhinology. 2011; 49: 304-308.

11. Van Spronsen E, Ebbens FA, Fokkens WJ Normal peak nasal inspiratory flow rate values in healthy children aged 6 to 11 years in the Netherlands. Rhinology. 2012; 50: 22-25.

12. Thulesius $\mathrm{HL}$, Cervin A, Jessen $M$. The importance of side difference in nasal obstruction and rhinomanometry: a retrospective correlation of symptoms and rhinomanometry in 1000 patients. Clin Otolaryngol. 2012; 37: 17-22.

13. Hopkins C, Gillett S, Slack R, Lund VJ,
Browne JP. Psychometric validity of the 22-item Sinonasal Outcome Test. Clin Otolaryngol. 2009; 34: 447-454.

14. Akaike H. A New Look at the Statistical Model Identification. IEEE transactions on automatic control. 1974; 19: 716-723.

15. Gregg I, Nunn AJ. Peak expiratory flow in normal subjects. Br Med J. 1973; 3: 282-284.

Giancarlo Ottaviano, MD, PhD

Department of Neurosciences

Otolaryngology Section

University of Padova

Via Giustiniani 2

35128 Padova

Italy

Tel. $+39(0) 498212029$

Fax: +39 (0)498213113

E-mail: giancarlo.ottaviano@unipd.it

\section{0th INTERNATIONAL COURSE IN ADVANCED SINUS SURGERY TECHNIQUES}

Dissection course with fresh frozen cadaver heads

Teacher of Honour:

Prof. Richard J Harvey

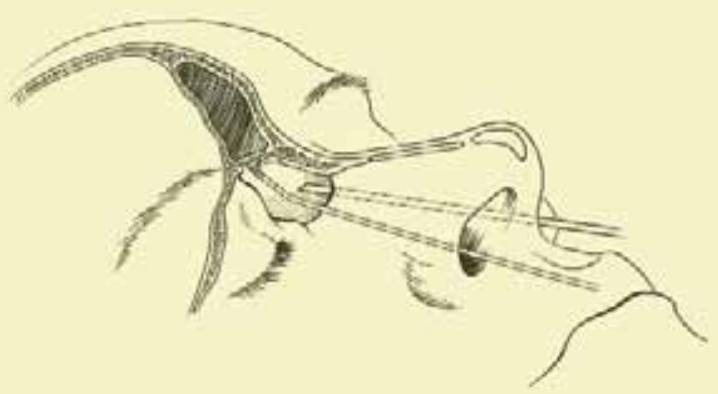

March 7-8, 2013

Department of Otorhinolaryngology

Academic Medical Center of the University

of Amsterdam

The Netherlands

www.sinuscourse.nl

For further information contact Wytske J. Fokkens, $M D, P h D$

ENT dept. AMC Course Secretariat

Tel: 00312056685 86 / Fax 0031205669573

Email:m.b.vanhuiden@amc.uva.nl 